\title{
Pancreatitis aguda secundaria a un trauma abdominal: reporte de caso de una niña de 7 años
}

\author{
Acute pancreatitis secondary to an abdominal trauma: case report of a 7-year-old child
}

\author{
Dra. Laura Montoya-Sáncheza , Dra. Rocío del P. Pereira-Ospina ${ }^{b}$, \\ Dr. Javier Y. Pinzón-Salamancac y Dr. José M. Suescún-Vargas ${ }^{d}$
}

\begin{abstract}
RESUMEN
La pancreatitis consiste en la inflamación aguda del páncreas, que se caracteriza, clínicamente, por dolor abdominal. Para realizar el diagnóstico, es necesario que se presente la elevación demarcadores bioquímicos, como amilasa o lipasa pancreáticas. Esta afección es la enfermedad pancreática más frecuente tanto en niños como en adultos. Su presentación en la población pediátrica está en aumento y tiene varias etiologías, como infecciones, trauma, intoxicación y enfermedades metabólicas. Entre los mecanismos de trauma, se han descrito maltrato infantil, caídas, trauma con el manubrio de la bicicleta y accidentes de tránsito.

En este artículo, se presenta un caso de pancreatitis secundaria a un trauma abdominal con el manubrio de una bicicleta en una paciente de 7 años.

Palabras clave: niño, pancreatitis aguda, trauma, pancreatitis, traumatismos abdominales.
\end{abstract}

\begin{abstract}
Pancreatitis consists in acute inflammation of the pancreas that is clinically characterized by abdominal pain. To make the diagnosis it is necessary the elevation of biochemical markers like pancreatic amylase or lipase. It is the most frequent pancreatic disease in both children and adults. Its presentation in the pediatric population is increasing and has several etiologies such as: infections, trauma, intoxication and metabolic diseases. Trauma mechanisms have been described: child maltreatment, falls, trauma with the bicycle handle and traffic accidents.

In this article, we present a case of pancreatitis secondary to an abdominal trauma with the handle of a bicycle in a patient of 7 years.

Key words: child, acute pancreatitis, trauma, pancreatitis, abdominal injuries.
\end{abstract}

a. Universidad del Rosario, Bogotá, Colombia.

b. Servicio Social Obligatorio de Investigación Pediátrica del Instituto Roosevelt.

Universidad del Rosario, Bogotá, Colombia.

c. Instituto Roosevelt.

Universidad del Rosario, Andes y Sabana, Bogotá, Colombia.

d. Instituto Roosevelt.

Escuela de Medicina y Ciencias de la Salud, Universidad del Rosario, Andes y Sabana, Bogotá, Colombia.

Correspondencia:

Dra. Rocío del P. Pereira Ospina: rpereira@ioir.org.co.

Financiamiento: Ninguno.

Conflicto de intereses: Ninguno que declarar.

Recibido: 22-6-2017

Aceptado: 25-9-2017 http: / / dx.doi.org/10.5546/aap.2018.e308

Cómo citar: Montoya-Sánchez L, Pereira-Ospina RP, PinzónSalamanca JY, et al. Pancreatitis aguda secundaria a un trauma abdominal: reporte de caso de una niña de 7 años. Arch Argent Pediatr 2018;116(2):e308-e311.

\section{INTRODUCCIÓN}

La pancreatitis aguda se define como la presencia histológica de un proceso inflamatorio agudo reversible dentro del páncreas. El cuadro clínico se caracteriza por dolor abdominal y niveles elevados de enzimas pancreáticas. ${ }^{1}$

Para realizar el diagnóstico, se deben cumplir, al menos, dos de los siguientes criterios:

1. Dolor abdominal sugestivo de pancreatitis aguda o compatible con ella (de inicio agudo y más frecuente en el epigastrio).

2. Niveles de amilasa o lipasa tres veces superiores al normal (IU/1).

3. Hallazgos imagenológicos compatibles con pancreatitis aguda. ${ }^{2,3}$

La incidencia de la pancreatitis aguda en la población pediátrica ha ido en aumento en Estados Unidos y es de 3,6-13,2 casos por 100000 niños por año. ${ }^{4,5}$ Son varias las causas de pancreatitis en niños; entre las de mayor frecuencia, se encuentran enfermedades sistémicas, como sepsis, shock, enfermedad inflamatoria intestinal, síndrome urémico hemolítico (10-50\%); medicamentos, como ácido valproico, 6-mercaptopurina/ azatioprina, 1-asparaginasa, masalamina, trimetoprimasulfametoxazol, furosemida, tacrolimus, esteroides (5-25\%); factores obstructivos: cálculos biliares, páncreas divisum, quistes en el colédoco, disfunción del esfínter de Oddi, páncreas anular (10-30\%); trauma (10-20\%); infección viral (8-10\%); enfermedades metabólicas, como cetoacidosis diabética, hipertrigliceridemia, errores innatos del metabolismo e hipercalcemia (5-10\%); y, finalmente, causas idiopáticas (15-30\%). ${ }^{6}$ 


\section{REPORTE DE CASO}

Paciente femenina de 7 años de edad natural y procedente de Madrid (Cundinamarca), sin antecedentes de importancia. Ingresó a un hospital de tercer nivel por un cuadro clínico de 2 días de evolución consistente en dolor abdominal de leve a moderado, asociado a emesis en múltiples ocasiones, disminución de deposiciones y coluria. La sintomatología se inició luego de un trauma abdominal cerrado de moderado impacto con el manubrio de la bicicleta con la que jugaba dos días antes. Consultó al lugar de remisión, donde tomaron exámenes paraclínicos, que documentaron elevación importante de amilasa (1213 U/L), hemograma con leucocitosis y neutrofilia y función renal conservada. Allí, lo manejaron con líquidos endovenosos abundantes, analgesia con antiinflamatorios no esteroideos (AINE) y la remitieron con el diagnóstico de pancreatitis aguda para continuar su manejo.

$\mathrm{Al}$ ingreso, la paciente tenía aceptable estado general, alerta, afebril, con deshidratación grado ii, sin dificultad respiratoria. Al momento del examen físico, con abdomen blando, no distendido, doloroso ante la palpación profunda con predominio en el epigastrio, sin signos de irritación peritoneal, peristaltismo presente. Adecuada perfusión distal, sin signos de bajo gasto.

Los exámenes paraclínicos de control al ingresar a la institución mostraron amilasa (4530 U/L) y lipasa (4681 U/L) aumentadas más de tres veces del valor normal, bilirrubinas y transaminasas dentro de los valores normales, leucocitosis con neutrofilia, colesterol y electrolitos dentro de los límites normales. La ecografía abdominal y pélvica evidenció escasa cantidad de líquido perihepático, vesícula biliar distendida de paredes delgadas sin cálculos en su interior, vía intra- y extrahepática de calibre normal.

La gravedad de la pancreatitis fue clasificada como Apache 4, Ranson 1, por lo cual la paciente fue trasladada a la Unidad de Cuidado Intensivo Pediátrico (UCIP). Se ampliaron los estudios con una tomografía axial computarizada (TAC), que mostró un gran aumento del tamaño del páncreas con realce tras la administración del medio de contraste, sin evidencia de colecciones peripancreáticas ni formaciones pseudoquísticas, y se catalogó como pancreatitis aguda Balthazar B,

\section{Figura 1. Tomografía axial computarizada abdominal}

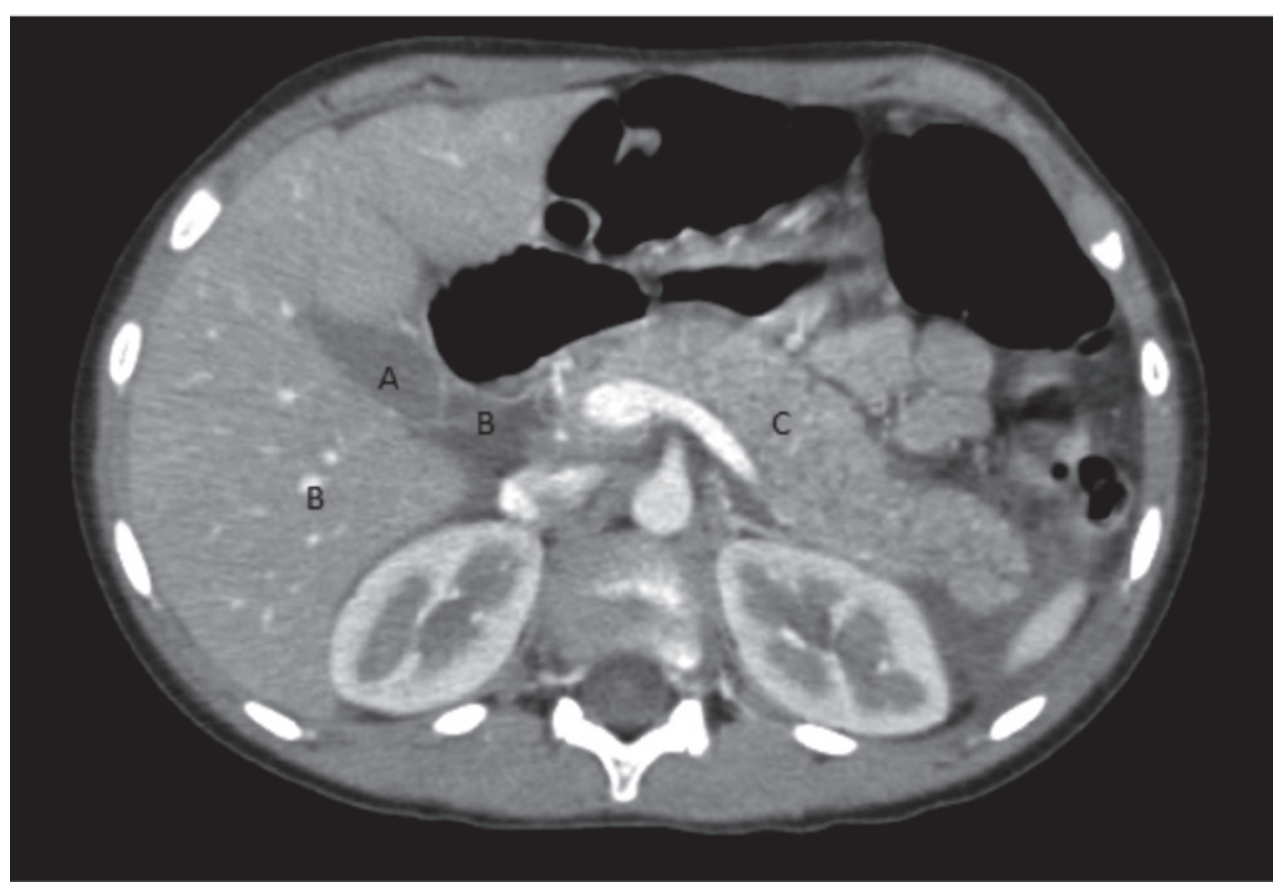

(A) La vesícula biliar se encuentra distendida, con paredes no engrosadas, sin evidencia de cálculos en su interior.

(B) La vía biliar intra- y extrahepática es de calibre usual. (C) El páncreas de encuentra aumentado de tamaño y se realza con el medio de contraste. No hubo evidencia de edema peripancreático, colecciones peripancreáticas o formaciones pseudoquísticas, hallazgos que se encuentran en relación con la pancreatitis aguda clasificación Balthazar B. 
con la vía biliar intra- y extrahepática sin lesiones aparentes (Figura 1).

Durante su estancia en la UCIP, se dio inicio a la nutrición parenteral total, dado que presentó hipoglicemia sintomática. Se dejó en reposo intestinal por cinco días y, posteriormente, se decidió reiniciar por vía oral, con adecuada tolerancia. Por la apropiada evolución clínica, se trasladó a la sala y, luego de siete días de hospitalización, presentó una mejora significativa de los síntomas, cesó el dolor abdominal, toleró adecuadamente la vía oral y egresó en buenas condiciones generales.

\section{DISCUSIÓN}

Varios estudios han identificado un aumento en la incidencia de la pancreatitis aguda en la población pediátrica. Sin embargo, no está clara la razón de este ascenso en el número de casos; puede estar relacionado con un mejor conocimiento de esta patología por parte del personal médico. ${ }^{7-9}$

Entre las manifestaciones clínicas de pancreatitis aguda, se encuentran dolor abdominal, que es uno de los síntomas más tempranos en niños, fiebre, emesis, ictericia, dolor de espalda, acolia, diarrea y pérdida de la conciencia. En el rango de edad en el que se encuentra nuestra paciente, los síntomas más comunes son dolor abdominal y fiebre acompañados de episodios eméticos. ${ }^{10}$ La paciente consultó por dolor abdominal, episodios eméticos, disminución en el número de deposiciones y coluria, que son síntomas frecuentes en esta patología.

Los hallazgos imagenológicos que se pueden encontrar en la pancreatitis son ascitis, hemorragia, necrosis del parénquima y / o grasa pancreáticas, edema y colecciones peripancreáticas o pancreáticas. ${ }^{3}$ En pacientes pediátricos, el estudio imagenológico de elección es el ultrasonido, debido a la poca grasa corporal y el pequeño tamaño corporal de los niños. Entre sus ventajas, se encuentran su buena disponibilidad en los servicios de salud, la falta de radiaciones ionizantes y que no requiere sedación. La TAC abdominal es más sensible para detectar pancreatitis y gradúa la gravedad de la patología; sin embargo, una de sus desventajas es el uso de radiación ionizante. La resonancia magnética (RM) tiene mayor resolución que la TAC y permite detectar la inflamación pancreática en estadios más tempranos. ${ }^{3} \mathrm{~A}$ la paciente se le realizó una ecografía abdominal, que reportó la ausencia de colecistitis y colelitiasis. No se logró observar adecuadamente el páncreas por interposición de asas, por lo que se solicitó la TAC abdominal, cuyo hallazgo fue el aumento del tamaño del páncreas compatible con la clasificación Balthazar B.

Aproximadamente, el 8-20\% de las pancreatitis son secundarias a un trauma, es decir, es una causa poco frecuente de pancreatitis en la población pediátrica. ${ }^{6-11}$ En el estudio realizado por Park y colaboradores, se identificó que los niños menores de dos años presentaban menos signos y síntomas comparados con niños de mayor edad. Además, la lipasa es más sensible que la amilasa sérica para realizar el diagnóstico en todas las edades pediátricas. ${ }^{12}$ En nuestro caso, a la paciente se le realizaron pruebas de amilasa y lipasa séricas, las cuales superaron tres veces el valor normal.

Para el manejo del trauma pancreático, se debe descartar el compromiso de otros órganos. La laparotomía está indicada en el caso de compromiso multiorgánico, que incluiría la reparación del páncreas. El manejo conservador se debe instaurar en pacientes con contusión o laceraciones superficiales del páncreas. ${ }^{13,14}$ Este tratamiento consiste en aliviar el dolor abdominal, corregir la deshidratación, prevenir las complicaciones y garantizar una adecuada nutrición. ${ }^{5}$ La paciente no requirió el manejo quirúrgico, ya que no se encontraron disrupciones del tejido pancreático.

El 85\% de las pancreatitis son intersticiales y el restante son necrotizantes. La mortalidad de la pancreatitis es del 3\% en las intersticiales; del $17 \%$ en las necrotizantes; en ausencia de falla orgánica, es 0 ; con afección de un solo órgano, es del $3 \%$; y, con falla multisistémica, es del $47 \%$. En el caso de la paciente, no hubo falla orgánica; hubo una adecuada reanimación con líquidos, reposo intestinal, soporte nutricional y se trasladó a la UCIP para el monitoreo estricto, por lo cual el pronóstico fue favorable. ${ }^{15}$

\section{REFERENCIAS}

1. Rodríguez Schulz D, Martínez A, Guzmán MB, et al. Pancreatitis aguda grave e infección por el virus de la influenza A (H1N1) en un paciente pediátrico : reporte de caso. Arch Argent Pediatr 2015;113(4):e215-8.

2. Sathiyasekaran M, Biradar V, Ramaswamy G, et al. Pancreatitis in Children. Indian J Pediatr 2016;83(12-13):145972.

3. Restrepo R, Hagerott HE, Kulkarni S, et al. Acute Pancreatitis in Pediatric Patients: Demographics, Etiology, and Diagnostic Imaging. AJR Am J Roentgenol 2016; 206(3):632-44. 
4. Muñoz Lozón AM, Iglesias Blázquez C, Menéndez Arias C, et al. Pancreatitis necrohemorrágica en un niño de 11 años. Caso clínico. Arch Argent Pediatr 2015;113(1):e39-42.

5. Kramer C, Jeffery A. Pancreatitis in children. Crit Care Nurse 2014;34(4):43-52.

6. PohlJF, UcA.Paediatric pancreatitis. Curr Opin Gastroenterol 2015;31(5):380-6.

7. Morinville VD, Barmada MM, Lowe ME. Increasing incidence of acute pancreatitis at an American pediatric tertiary care center: is greater awareness among physicians responsible? Pancreas 2010;39(1):5-8.

8. Nydegger A, Heine RG, Ranuh R, et al. Changing incidence of acute pancreatitis: 10-year experience at the Royal Children's Hospital, Melbourne. J Gastroenterol Hepatol 2007;22(8):1313-6.

9. Lopez MJ. The changing incidence of acute pancreatitis in children: a single-institution perspective. J Pediatr 2002;140(5):622-4.
10. Suzuki M, Sai JK, Shimizu T. Acute pancreatitis in children and adolescents. World J Gastrointest Pathophysiol 2014;5(4):416-26.

11. Kandula L, Lowe ME. Etiology and outcome of acute pancreatitis in infants and toddlers. J Pediatr 2008;152(1):10610.e1.

12. Park AJ, Latif SU, Ahmad MU, et al. A Comparison of Presentation and Management Trends in Acute Pancreatitis Between Infants/Toddlers and Older Children. J Pediatr Gastroenterol Nutr 2010;51(2):167-70.

13. Debi U, Kaur R, Prasad KK, et al. Pancreatic trauma: A concise review. World J Gastroenterol 2013;19(47):9003-11.

14. DixitP, SharmaV,SinghKR, etal.Conservative management of a case of traumatic pancreatitis in childhood: a case report. Eur Rev Med Pharmacol Sci 2014;18(12):1687-9.

15. Banks PA, Freeman ML. Practice guidelines in acute pancreatitis. Am J Gastroenterol 2006;101(10):2379-400. 